\title{
EUROPEANA AND 3D
}

\author{
Daniel Pletinckx \\ Visual Dimension bvba, Lijnwaadmarkt 47, 9700 Ename, Belgium \\ daniel.pletinckx@visualdimension.be
}

KEY WORDS: Europeana, 3D, PDF, interactivity, 3D publishing, resource exploration, explorative interface, learning objects

\begin{abstract}
:
The current 3D hype creates a lot of interest in 3D. People go to 3D movies, but are we ready to use 3D in our homes, in our offices, in our communication? Are we ready to deliver real 3D to a general public and use interactive 3D in a meaningful way to enjoy, learn, communicate? The CARARE project is realising this for the moment in the domain of monuments and archaeology, so that real 3D of archaeological sites and European monuments will be available to the general public by 2012 .

There are several aspects to this endeavour. First of all is the technical aspect of flawlessly delivering 3D content over all platforms and operating systems, without installing software. We have currently a working solution in PDF, but HTML5 will probably be the future. Secondly, there is still little knowledge on how to create 3D learning objects, 3D tourist information or 3D scholarly communication. We are still in a prototype phase when it comes to integrate 3D objects in physical or virtual museums. Nevertheless, Europeana has a tremendous potential as a multi-facetted virtual museum. Finally, 3D has a large potential to act as a hub of information, linking to related 2D imagery, texts, video, sound. We describe how to create such rich, explorable 3D objects that can be used intuitively by the generic Europeana user and what metadata is needed to support the semantic linking.
\end{abstract}

\section{INTRODUCTION}

Europeana is the European Digital Library for Culture. It is structured as a portal with the content itself residing on national servers. By using a metadata standard (EDM) for all European organisations that contribute to Europeana, this portal allows to search for cultural resources over all collections in Europe. One of the objectives of the European project CARARE is to integrate $3 \mathrm{D}$ representations and VR (virtual reality) of monuments, historical buildings and archaeology into Europeana for tourists, educational users, scholars and general public. The description of CARARE on the 'about' page of Europeana states: "CARARE makes a first step towards integration of a wide range of high quality, validated 3D/VR data in an international digital library/museum/archive context". CARARE will also be the first project to use the new EDM metadata standard that supersedes the older ESE metadata standard that was used during the start-up phase of Europeana.

\section{3D FOR THE LAYMAN}

CARARE focuses on representations of archaeological and architectural objects, so 3D/VR within CARARE refers to 3D representations of such objects. This includes 3D models of a monument or landscape or archaeological object as it survives in the present day through $3 \mathrm{D}$ digitisation by laser scanning, image modelling (Nilsson et al, 2007) or other techniques, reconstructions of such monuments (Pletinckx, 2007) or landscapes (Pletinckx, 2008) as they appeared at various times in the past through a manual virtual reconstruction process, or digital restorations of archaeological objects or monuments through editing of 3D digitised models (Müller, 2007). Each of these three forms of 3D has its own specific requirements and properties, which play an important role when we want to make these 3D models available to a general public.

When providing a 3D model instead of an image or movie of an object, we need to create a clear advantage for the user. This advantage can be for example that the complex structure of the building or object can be understood or appreciated better, through specific viewpoints or cross sections or alterations of the current situation. Another advantage is to visualise and experience spaces that are difficult to access such as caves, or simply don't exist anymore such as destroyed buildings or ancient landscapes which can be represented as virtual reconstructions.

In most cases however, 3D wants to be more than just visualisation only. 3D models can be used as an interactive user interface to attach information to objects, so that the 3D model becomes an explorative environment. If we virtually reconstruct a Roman villa, we may be clicking on a wall painting to see how it looks today, or click on a zone to find out how that room was used and by whom. We might like to see how the siege of a medieval castle developed, how the attacking party could breach or undermine walls or how the defending party could cope with attacks from the siege towers. But we might also want to know how archaeologists discover the past in analysing and comparing archaeological remains. Studies have shown that learning happens faster and better, with more persistent results, if it is based upon personal decisions and interaction at a personal pace. The richness of a 3D model allows storing much more information inside than an image, so that personal interaction creates a closer psychological bond with the subject than other media.

In other words, we need to use 3D when the visualisation, the animation and exploration capabilities of 3D help us to create added value through better understanding, better memorising or being more appealing. It is exactly this added value that makes interactive $3 \mathrm{D}$ outperform the current multimedia tools in tourism, education and research, hence make 3D visualisation worthwhile using in Europeana.

For meeting the goals of CARARE and Europeana, we are convinced that the focus of $3 \mathrm{D}$ should be on resource exploration, just like with the other formats (text, images, video and sound) that are currently available. This means that we consider the linking of information to the $3 \mathrm{D}$ model as most 
important, while autonomous and interactive behaviour are considered too far-fetched for the start-up phase of 3D in Europeana. This means also that aspects such as photorealism or high degree of detail are in fact of secondary order, while content, interactivity and linking to other content are the most important in this initial phase.

As we start however a long-term process of creating 3D resources for general and professional use in cultural heritage, we need to be sure that the choices we make now allow a further evolution and richer functionality in the near future. In other words, in the CARARE project, we need to focus on creating a first batch of about 3000 high quality 3D objects, and enabling the content providers to do in a coordinated and uniform way, without going too far in functionality and sophistication of the 3D objects (which can be implemented in a later stage, when the workflow is well defined and established). But we are convinced that the possibilities of the approach, as we outline here in this text, are far richer and potent than will be used in the CARARE project itself. Nevertheless, they will be taken into account for inclusion in workflow descriptions to allow deploying this extra functionality in the near future by any cultural heritage organisation that wants to publish $3 \mathrm{D}$ resources in Europeana.

In other words, 3D in Europeana means high quality data that allows a certain degree of interactivity and exploration capabilities (through different techniques). The difference with other repositories such as Google 3D Warehouse is the quality of the data (see chapter 9) and especially metadata (see chapter 8) that is guaranteed by the cultural heritage organisation that has created the 3D data. The metadata of the 3D model provides a rich context that helps the user to understand, assess and explore the object, and situate it within a larger historical and cultural framework.

\section{3D REQUIREMENTS}

Europeana requires that formats to deliver content are open and standardised, and can be sustained on the long term (this means in practice also that the format at least must be used by a sufficient number of users, otherwise the market will not provide the necessary tools to maintain the format or migrate to other formats).

But 3D is more than the format only. As visualising 3D is not trivial, we also need to state requirements for the 3D visualisation process. For maximising the number of users that can be served, we need to require that the $3 \mathrm{D}$ viewer is available on all major platforms (Windows, Mac OS, Linux) and is free.

For maximum user comfort when using 3D, we require also that the $3 \mathrm{D}$ viewer is already present on nearly all computers (in other words, has a high market penetration) and is available within all major browsers. In other words, most people will not have to install software or plug-ins.

For maximum sustainability, we also require that the $3 \mathrm{D}$ viewer has a proven track record, is mature in functionality and is provided by multiple companies.

Europeana focuses on resource exploration. This means that the 3D viewer needs to support URLs and links to other documents, to provide linking to other resources and allow continued exploration. This also means that the $3 \mathrm{D}$ viewer needs ways to embed text and images to allow the users to understand the 3D they are visualising and exploring.

Besides the general public, CARARE focuses mainly on domains such as education, tourism and research. This means that the $3 \mathrm{D}$ visualisation process needs to have functionalities that support use in these domains.

As we need to support the concept that Europeana only refers to a $3 \mathrm{D}$ resource, while showing a thumbnail representation, the real $3 \mathrm{D}$ data sits on the national servers of the aggregators. This means that we need to come up with a solution that is to a certain extent standardised and uniform on all national resource servers, so that the user does not get confused or bored.

\section{PDF AS 3D FORMAT}

When analysing all currently available 3D viewers, there is only one technology at this moment that matches nearly all of these requirements, which is PDF. Since 2005 (Acrobat version 7), PDF has been extended with 3D capabilities. Currently we have Acrobat version 10 and 6 years of maturing technology.

PDF definitely has a proven track record. PDF (Portable Document Format) was developed in 1993 and is the de facto standard for exchange of digital documents and forms. It is also an open format since the full PDF format (version 1.7) has been accepted in 2008 by the International Organisation for Standardisation as standard ISO 32000-1. This ISO standard does include the 3D capabilities of PDF. Extensions to this standard are open and published, the Acrobat version 9.1 for example produces PDFs with extension level 5. The goal of this standardisation is of course to ensure long term preservation of PDF documents. An update of the ISO standard (i.e. ISO 32000-2) that includes these extensions has been accepted (also known as PDF version 2.0). Having PDF as an ISO standard means that third parties can develop applications that read or write PDF without having to pay royalties to Adobe. Several companies are already providing such applications and 2010 has seen several new companies and applications emerge, which is very good news for the long-term support of PDF as a format and a technology.

PDF readers are available on all major platforms (Windows, Mac OS, Linux) and are available for free. Adobe, the company that created PDF, claims that its Acrobat PDF reader is present on $89 \%$ of all computers. This number is considered by experts to be accurate. The reason that this number is not higher is probably that Apple provides an alternative image and PDF viewer (called Preview) that is preinstalled. For Windows, most computers come with the Acrobat PDF reader preinstalled, so most people don't have to bother to install a PDF reader. As Preview does not visualise the 3D parts of a PDF file, users need to install the free Acrobat Reader on Mac platforms. Preview in fact has some older PDF authoring capacities (extracting pages, merging PDFs, encrypting, ...) but lacks the more recent functionality including visualisation of 3D. Preview on the other hand has functionality to visualise Collada 3D files (for example from Google 3D Warehouse) directly.

3D PDF functionality has been integrated in Acrobat Reader since 2005. This means that even older computers, that haven't been updated since years, are capable of displaying 3D PDF, as long as we limit ourselves to the basic 3D PDF functionality that is supported by that version (PDF version 1.6). To deal with older computers, we could create Acrobat version 7 compatible 3D PDF files, which is in most cases not penalising 
at all. To comply with the ISO 32000-1 standard however, we recommend to create PDFs that are compatible with Acrobat version 8 (PDF version 1.7) that has been released in 2006.

3D PDF can be visualised within most browsers on most platforms. The recent PDF plug-in for Mac OS X Intel for Firefox, provided by Mozilla, however does not support 3D yet. In this aspect, PDF fails to a certain extent, but there is simply no alternative today and most people download PDF files anyway instead of opening them in their browser, so this noncompliance to the requirements above does not look to have severe drawbacks.

PDF supports all European languages, and much more. This looks obvious, but the language support of 3D applications has always been very weak, and until today, strange behaviour or bugs occur in most 3D applications when using non-ASCII texts, part names or descriptions.

Since the end of 2010, Adobe has moved its 3D development to a separate company, called Tetra 4D. As this company is a cooperation with Tech Soft 3D, a specialised 3D company with a long track record, this move allows a much more focused development for and support of the different market segments that use 3D PDF. This needs to be seen as a very positive move and marks a transition for 3D PDF from "early adopter phase" to "mainstream product". The appearance in the last years of good third party software for the authoring of 3D PDF also can be seen as a positive sign of a maturing technology. Assigning 3D PDF as the platform for 3D in Europeana should fit perfectly in this evolution.

The 3D PDF creation process deals with most available 3D file formats resulting from both digitisation and virtual reconstruction processes. Internally, the $3 \mathrm{D}$ model within the PDF is stored in a U3D or PRC format. U3D is the Universal 3D file format, standardised as ECMA-363 by ECMA International, the (American) industry association founded in 1961 dedicated to the standardization of Information and Communication Technology (ICT) and Consumer Electronics (CE). PRC (Product Representation Compact) has been developed by Adobe to provide a compact and versatile format for $\mathrm{CAD} / \mathrm{CAM} / \mathrm{CAE}$ data (for the domains of computer aided design, manufacturing and engineering). $\mathrm{PRC}$ is an open format that realises high compression rates for CAD/CAM/CAE data and is in the process of becoming an ISO standard for such data.

For cultural heritage purposes, U3D however is the best choice. Tests on many cultural heritage $3 \mathrm{D}$ files have shown that the compression capabilities of U3D typically better than PRC (over 4 case studies, U3D yielded a file reduction to $46,2 \%$ of the original file size against $77,2 \%$ for PRC), while U3D provides better possibilities for animation. Currently, 3D PDF uses U3D version 3 (U3D version 4 is available since June 2007).

\section{3D WORKFLOW}

Not all of the 3D resources, that are created today within the context of cultural heritage organisations, are suited as such for use in Europeana, where a published item resides as a single object, armed with the appropriate metadata to be discovered and semantically linked to related objects. This problem arises from three major issues.
A first issue is that a 3D resource, unlike most other Europeana resources, can be explored in itself, so it provides other exploration mechanisms than the typical resource exploration mechanisms that are present and planned in Europeana. Exploration is a natural, intuitive thing to do with $3 \mathrm{D}$ sites and buildings, so we should not put his opportunity aside.

A second issue is that most 3D has a high 'information load', in other words, a lot of information is connected to the 3D resource, much more than to a typical Europeana resource. So we need to provide easy and intuitive ways to find this information, linked to the natural exploration possibilities that a $3 \mathrm{D}$ resource provides.

A third issue is that most 3D resources, that represent spaces, are in fact not one object but a collection of highly interconnected parts that each have specific information. Even if the object is perceived as one single object, we currently don't have the technical framework within Europeana to attach information to different spatial regions of the 3D resource, so we would be forced to divide the object into different components that each have specific metadata. But for aesthetical and conceptual reasons, we don't want to divide the 3D resource into separate objects, each with their specific metadata as we would do with museum objects, belonging to a museum collection. So we rather want to establish mechanisms that allow resource exploration within the 3D model, so that the 3D model can remain undivided and preserve its nature as representation of a space.

In other words, the design of resource exploration for 3D Europeana objects, especially if they represent buildings and landscapes, needs to allow a part of the resource exploration to happen within the $3 \mathrm{D}$ resource, in addition to the resource exploration mechanisms, based upon metadata, outside of the object. In other words, a small part of the content metadata needs to be integrated within the $3 \mathrm{D}$ resource, to allow an intuitive $3 \mathrm{D}$ resource exploration that preserves the nature of the $3 \mathrm{D}$ resource.

The balance between the outside and inside metadata depends on the nature of the $3 \mathrm{D}$ resource and on the skills of the creating organisation to integrate some of the metadata in the interaction design. For archaeological museum objects, all of the metadata can be outside the 3D resource. In other words, the 3D resource only requires interactive visualisation from all sides, like you would have the object in your hand. For buildings, archaeological sites, man-made structures and landscapes, we need to integrate some of the content metadata into the 3D resource to support resource exploration within the $3 \mathrm{D}$ resource.

Practically, this means we end up with two types of 3D PDF, one for objects and one for spaces. The object one can be seen as a pure file format conversion from the original 3D file format to $\mathrm{PDF}$ as a 3D visualisation tool, where the provided metadata is sufficient to identify the object, learn about it and find related objects or context information. A PDF that shows spaces (buildings, archaeological sites, man-made structures, landscapes) will be structured as a text document, containing one or more $3 \mathrm{D}$ windows plus some text, images or URLs that help to explore and understand the 3D data.

In the first case (objects), the visualisation capabilities of PDF are in most cases sufficient for interacting with such objects. In the creation of the PDF, standard views such as front, left, right, top, back, ... can be added automatically for easier evaluation of the object. The added value of the $3 \mathrm{D}$ is the interactive 
visualisation of the object from all sides, like you would have it in your hand. This is certainly an added value as most archaeological objects cannot be viewed like this, and some are even not on display at all. The example below shows a museum object, when clicked, the object can be inspected from all sides, while the toolbar allows to visualise (orthographic) standard views (top, left, front,...) that have been created automatically at file import.

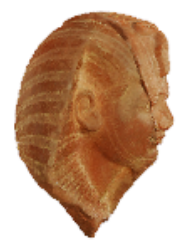

Figure 1. 3D model of pharaoh head (Allard Pierson Museum)

In the case of museum objects, we can - when appropriate also use the available 3D PDF authoring tools in the form of software libraries (PDF3D) to add some metadata (title, short description, basic parameters or information) automatically in the PDF. In this way, the PDF is not only a nice 3D visualisation tool but the PDF file also becomes a useful standalone resource that can be printed, shared for educational use amongst students or taken to field on a laptop by archaeologists. For example, the CATA archaeological reference collection of Iberian pottery (CATA) can be automatically turned into a set of one page PDFs, each containing a 3D model of a certain vessel, together with some basic information that is taken automatically from the database.

In the second case (spaces), it is sufficient to add a few paragraphs of text and a few images to the $3 \mathrm{D}$ to create the appropriate exploration mechanisms. More precisely, PDF authoring allows to link keywords in the text to specific views in the 3D resource, so that clicking on those keywords produces the right view in the $3 \mathrm{D}$ window. This simple VR behaviour is easy to author but provides a very powerful navigation tool with a very low user threshold, as demonstrated in the example below (click the words in blue).

This example shows a virtual reconstruction of the church and abbey of Saint John Evangelist in Biograd, Croatia. This Benedictine abbey consisted of a large Romanesque church with an extended choir for the monks and some abbey buildings around a cloister garden: an upstairs dormitory, the chapter room in which daily meetings were organised, the kitchen with adjacent storage room and the refectory where the monks took their meals after washing their hands at the well.

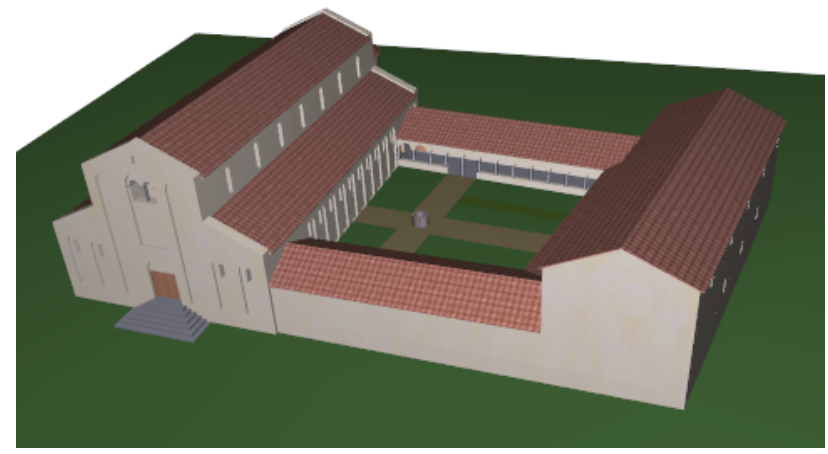

Figure 2. 3D virtual reconstruction of Saint John abbey, Croatia
As you can notice, the small text above provided a fully guided tour through the 3D virtual reconstruction, without any further user interaction with the 3D, giving direct information about the structure of the abbey and the daily life of the monks. The user can of course explore the 3D model at free will (choose first 'walking' as interaction mode in the toolbar for easy navigation) or use different rendering modes or illumination. For example, changing the rendering mode to 'shaded illustration' reveals the layered structure of the church and the abbey buildings and the way they were constructed (through medieval scaffolding).

\section{PDF AUTHORING}

An important technical aspect is the file conversion to 3D PDF. Here we need to distinguish between the file format in which the 3D data has been originally made, the open file format for long-term preservation of this $3 \mathrm{D}$ data, the metadata and the $3 \mathrm{D}$ PDF file.

Good news is that the 3D PDF authoring process has a large variety of file formats that it can start from, both from the 3D digitisation, the $3 \mathrm{D}$ design and the $3 \mathrm{D}$ digital preservation domains. So we need to decide which format is available and most suited to convert to 3D PDF. There are however some issues here.

First of all, a few important file formats are currently missing from the list of potential input formats, such as X3D (which is a quite popular open format for 3D files). This has no technical background but has everything to do with the ongoing strategy game and alliances between the major 3D players. Good news is that $3 \mathrm{D}$ files can be imported from digitisation processes (which typically use vertex colours, and can be inputted through VRML) and creation processes (which typically use texture mapping, and can be inputted through formats such as Collada, OBJ, 3D Studio, ...). It is useful to test the conversion process before starting major 3D projects to get optimal results and easy workflows.

A second issue that is related to the choice of file format to convert from is the differences in coordinate system. 3D systems that originate in the domain of surveying and $\mathrm{CAD} / \mathrm{CAM} / \mathrm{CAE}$ use the convention that the horizontal plane is parallel to the XY-plane (in other words, the positive Zdirection is up). 3D systems that originate in computer graphics on the other hand consider the XZ-plane as horizontal (in other words, the positive Y-direction is up). PDF uses the first system, so the latter formats need a simple 90 degrees rotation around the $\mathrm{X}$-axis before conversion to PDF. It is important to observe the correct vertical direction in $3 \mathrm{D}$ PDF as some interactions modes (such as the 'walking' mode) only move the camera in the horizontal plane.

A third issue is the completeness of the $3 \mathrm{D}$ data when doing conversion to PDF. PDF does package all required information in the file itself, while most other 3D file formats do not store the required information in a single file but in a set of files: the shape information, the material information (which are sets of parameters for material definition, colour image files for texturing, grayscale images for bump mapping or displacement mapping), font files, .... Having such a set of files is fine in a production environment that generates images as output (stills, video, ...) but this system is very vulnerable. For example, some 3D packages use absolute path names for material files, which is a problem when transporting the files to another environment. As the conversion of the $3 \mathrm{D}$ data to PDF can 
happen on another computer than the one that generated the $3 \mathrm{D}$ data, it is necessary to check and validate the available 3D data. Once successfully converted to PDF, the user does not have to worry as PDF encapsulates all required information in the PDF file itself to ensure portability. Also, each PDF file does behave exactly the same on every computer, also for $3 \mathrm{D}$, as this is the key element of the portability concept that PDF realises.

A fourth issue is internal compression within the PDF file. Although we recommend using U3D, there is currently an issue with $3 \mathrm{D}$ digitisation files using vertex colours, for which no compression can be used with the U3D internal format (otherwise, colour information gets lost). If file size matters, PRC can be used as internal format as PRC compression does not harm the vertex colours.

Another important technical aspect is the resolution of the 'professional 3D file', which can be too high for use by the general public, as it exceeds their requirements and the capabilities of their computers and becomes too large to be transferred easily over internet. So the 3D resolution of the 'professional files' from digitisation efforts needs to be reduced to a level that is much smaller but still remains aesthetical and functional. More and more, this functionality is integrated in the 3D digitisation production workflow, as reduction of the number of polygons is one of the standard features of professional digitisation software, and it helps the 'professional' users at the cultural heritage organisation to use the $3 \mathrm{D}$ data, for example on their laptop. On the other hand, free open source solutions (such as MeshLab) are becoming available that have high-end texture fitting and polygon reduction algorithms that can do the job, as for Europeana 3D resources, we want to have a minimal polygon count with a high quality texture map.

A final technical aspect is the accessibility of the 3D data. In most cases, cultural heritage organisations don't want the 3D data that is stored in the 3D PDF to be used for other purposes than viewing by the Europeana users. As 3D data can be exported from the 3D PDF (this feature is used a lot in the engineering applications of PDF), this can be prohibited explicitly by setting the 3D status to 'read only' when creating the file.

\section{VISUALISING COMPLEX 3D MODELS}

3D PDF is however not the ultimate solution for all 3D aspirations that Europeana could have. There are some cases in which this solution falls short: when the object is too large or too complex, when we want or need to have higher quality of $3 D$ visualisation than provided by the typical PDF reader software, when we use special render software such as raytracers (to visualise glass, reflections, gems, ...), volume renderers (to show for example tomography data of museum objects) or landscape renderers (that show terrain, vegetation, bathymetry, ...), when we want to show evolution over long periods of time of building complexes, sites or landscapes, when we want to use advanced animation techniques such as the use of avatars or particle systems.

For example, if we reconstruct landscapes or large cityscapes (such as Rome Reborn, showing Rome in $320 \mathrm{AD}$, showing 7000 highly detailed buildings with shadow), the amount of 3D data that we want to visualise exceeds by far the capabilities of the computer of the average Europeana user.
In most of these cases mentioned above, we can fall back on image based visualisation techniques that allow the $3 \mathrm{D}$ content to be visualised interactively. In the past, one of the most popular applications to do this was QuickTime VR, which has been discontinued by Apple in 2010. QuickTime VR provides in fact only 'pseudo 3D' as it can show only pre-rendered still images from predefined points of view (object mode) or a panorama of an environment (there are two modes, a cylindrical panorama relies on one long image, a cubic panorama or 'bubble' relies on 6 square images, organised as a cube). For a QuickTime VR object, smart organisation of the images however can create great interactivity, with capabilities to link images, URLs, text and other objects. Although such an object nearly feels like interactive $3 \mathrm{D}$, it is simply based upon a large amount of pre-rendered images, hence it's only pseudo-3D.

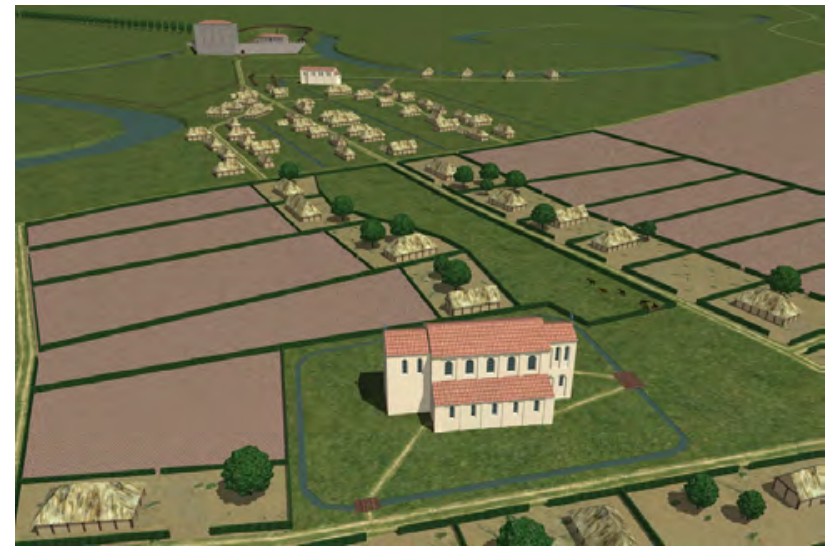

Figure 3. 3D virtual reconstruction of Ename in 1020

Currently, QuickTime VR functionality is being replaced by Flash and Java based solutions (Easypano, 2010) that provide the same possibilities as QuickTime VR (panoramas and VR objects). In most cases, we will need VR object functionality to provide the interactive exploration we want to achieve. If we want to show for example a digital restoration of a glass object that has be found in an excavation, we need to use a VR object or animation to visualise the glass properly, 3D PDF does not even comes close to a decent visualisation of glass objects. If we want to create $4 \mathrm{D}$ exploration (space plus time to show evolution) of a site or landscape (Pletinckx, 2008), we need to use a VR object as the amount of data will exceed by far the capabilities of 3D PDF. For example, the 4D visualisation of Ename, Belgium (see figure 3), that shows the evolution of the complete village from 1020 until 2004 through 12 historical periods, represents in total over $1 \mathrm{~GB}$ of $3 \mathrm{D}$ data and 800 hours of rendering time (as vegetation and shadows are used), so a VR object with pre-rendered imagery is the only way to visualise this interactively.

There are several possibilities to visualise such VR objects, one of the more recent possibilities is to have such panoramas and $V R$ objects integrated in a PDF file. When visualising complex animations (for example with avatars), we currently need to fall back on animated sequences in the supported video formats. Animations are always an alternative for visualising 3D but lack of course interactivity. This means concretely we can cover all $3 D$ visualisation in Europeana through PDF and video files. Because QTVR is no longer offered as functionality within QuickTime, we recommend not to create QTVR files anymore, but to use PDF and supported video-formats instead. 


\section{METADATA}

But there is more than the 3D data and the content only. On one hand, there is a lot of related information that needs to go into the metadata that gets attached to the 3D PDF file. On the other hand, some of the metadata can be duplicated in the 3D resource itself for better exploration (as explained in chapter 5).

Metadata for 3D is still very much a research domain, which is in development, especially for virtual reconstructions. Also, cultural heritage resources in real 3D for a general public are brand new, so we need to go through a process of validating the current 3D metadata insights.

The CARARE metadata scheme, which has been developed in close consultation with the partners that are involved in the $3 \mathrm{D}$ strand of CARARE, distinguishes clearly between the metadata for the 'heritage asset' (object, building , landscape...) and for the 'digital resource' (the 3D PDF) which is linked to the heritage asset. Most 3D content providers not only deliver 3D data but also a lot of other resources, such as related images, video, excavation reports, ... It is important that the right relationships are established between these elements so that old engravings of historical buildings or excavation photographs of its remains can be linked for example to a $3 \mathrm{D}$ virtual reconstruction, or that a museum object can be linked to the site where it has been found, with the archaeological site and the museum object are both represented as 3D model. The proposed CARARE metadata scheme allows establishing these relationships, except for the exact place of certain information within the 3D model, but this can be easily be solved by linking this information as images or text to specific views in the 3D model (as explained in chapter 5).

Concerning metadata, it is also important to highlight that the concept of 'collection' is also present in 3D objects representing spaces, with the additional requirement that a visualisation of the full space is provided. For example, if we visualise the Forum of Pompeii, we will need an overview 3D object plus a set of 3D PDF files, one per building on the Forum. It is obvious that the overview $3 \mathrm{D}$ object needs to link to each of the 3D PDFs that show the buildings, but also to 2D historical iconography that depicts the ruins in the $18^{\text {th }}$ and $19^{\text {th }}$ century. These links need to be represented by the metadata, that is attached to the overview $3 \mathrm{D}$ object and to the $3 \mathrm{D}$ resources that present the Forum buildings, but also by some links or compositions in the 3D objects themselves (see figure 5).

For the resource exploration of virtual reconstructions, it is even more important to link the different sources that were used to create the virtual 3D model, so that it becomes clear how the virtual model has been derived from the sources (see figure 4).

One important piece of information that also links digitised and reconstructed $3 \mathrm{D}$ models to the real world is the geographical information. Unfortunately, most archaeological information is quite sloppy about the height coordinate and the orientation, so it could require some manual intervention in the aggregation phase to get this information correct.

Putting some metadata also in the PDF itself would make the 3D resources more suitable for use as standalone resources (for example, a teacher providing Europeana 3D PDFs to the students for study and homework).

We are convinced that the creation of metadata needs to be part of the $3 \mathrm{D}$ data creation process within the cultural heritage organisation, which is completely focused on the professional use of those 3D files. This metadata then gets mapped (hence reduced) into the CARARE repository. Mapping towards Europeana will cause another reduction of the metadata. In other words, the only thing we need to do is giving directions towards the data providers for capturing the required metadata in their internal 3D creation process, so that the required metadata can be present at CARARE and Europeana level.

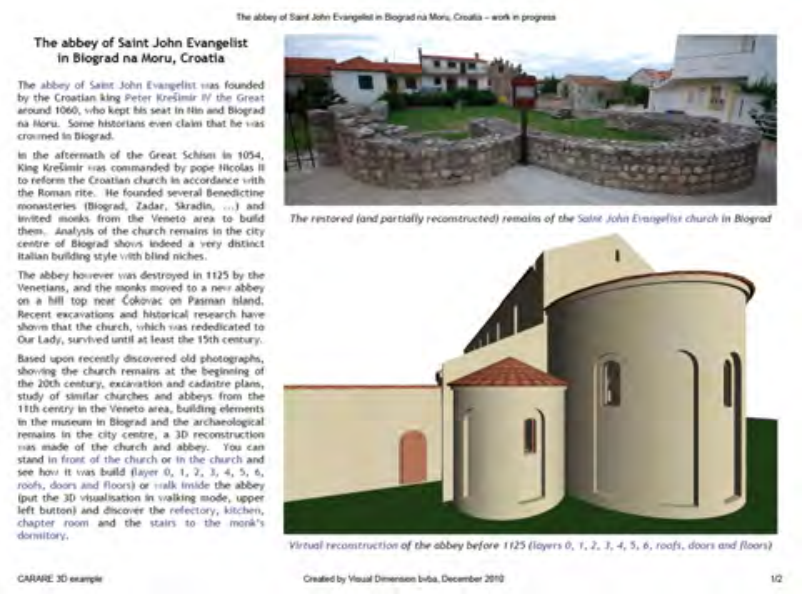

Figure 4. Screenshot of 3D PDF

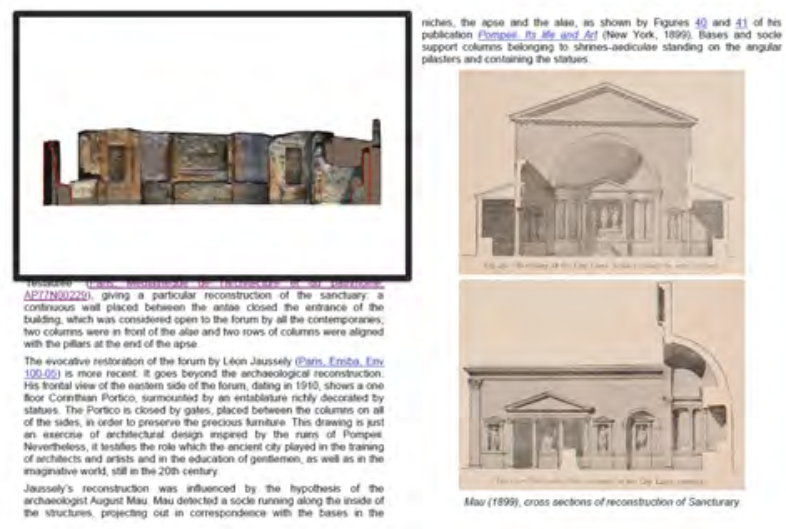

Figure 5. Screenshot of 3D PDF with floating window

\section{QUALITY}

One of the characteristics that sets apart Europeana from other resources is quality. We need to see $3 \mathrm{D}$ not as technological gimmick but as an opportunity to deliver content in a better and more efficient way. This means that, like in many innovative creation processes, we need to put "content first", hence focus on the quality and effectiveness of the 3D resources we create. Studies have shown that in many cases, photorealism and nice visualisation are only secondary to the perceived quality, which has more to do with 'presence', 'learning processes' and 'enjoyment'.

This means that we need to build in validation processes into the 3D creation workflow to safeguard the scientific accuracy of the $3 \mathrm{D}$ data and the provided context information, and the completeness of the required files to ensure a correct and optimal visualisation and interactivity and to maximise the opportunities for exploration, education and enjoyment. Further validation procedures needs to be performed on correctness and completeness of the associated metadata and 
on translations of the resource in different languages, if provided.

We need however to realise that an important part of the 3D data, that will be made available through Europeana, is legacy data, existing data that has been archived and in which the link with the creators can be broken or for which no funding is available to update the data. Therefore we need a validation procedure that checks the $3 \mathrm{D}$ data, that verifies if the data (as a whole or parts of it) have the required quality level, that defines the actions to be taken if the quality level is insufficient, and that plans the additional processing of the data before harvesting (making it multilingual, translating it in up to date file formats, establishing long-term digital preservation, ...).

Finally, there is also the marketing side of the story. 3D in Europeana needs to differentiate itself from Google 3D Warehouse and many other free 3D repositories by its quality and depth of content, which are obtained through this validation process and the know-how of the content provider.

\section{EVOLVING TECHNOLOGY}

It also needs to be clear that the current 3D hype will produce new visualisation opportunities in the near future that will comply with the requirements stated above. HTML5, that is expected to reach W3C (World Wide Web Consortium) Candidate Recommendation in 2012 does promise much better $3 \mathrm{D}$ rendering within browsers, the $3 \mathrm{D}$ part would be based upon the ISO-standard X3D. As the games world is very interested to have this (AppStorm, 2010), there is quite a pressure on the market to get this up and running. On the other hand, other players are trying not to loose their market share. Adobe is currently extending its Flash technology with 3D capabilities, but announced also to support HTML5. We need to see where the market will go to. The battle has definitely started, for example Apple refusing Flash technology on its platforms for many years could result from this HTML5 battle behind the scenes. Within one year, the situation probably will be much clearer. Currently, there are already good solutions on the market (for example Unity3D) that are multiplatform (including mobile devices) and provide great functionality, even including avatars, but they do not have the open and standard character that Europeana requires.

It would be great to have in Europeana interactive, intelligent walk-trough of virtual scenes or digitised buildings, as an avatar with other non-player characters showing how people lived and worked, like implemented for example in the Via Flaminia application (VHLab, 2007) in the National museum in Rome. However, the only fully reliable and deployed 3D environment that we can use today is 3D PDF. More sophisticated 3D visualisation tools will soon become available and maybe they will make it into Europeana.

Another major evolution that we see happening today is that the use of digital files in digital form is finally maturing. The availability of platforms such as the iPad and iPhone has dramatically improved the way we deal with digital data. A few years ago, a PDF file was still seen as suitable for printing only, now we start learning how to use it in a digital form, to read, discuss, exchange ideas and remarks, present, learn... The use of PDF files in for example education will dramatically change once teachers and students learn to use them in a digital form, including new capabilities such as 3D.
PDF (and especially its 3D capability) has already carved out some interesting digital niches such as training, digital manuals, engineering. We are convinced that PDF resources have a major future in a wide range of domains such as education, tourism and cultural heritage research. In other words, we are convinced that the choice of PDF as a cultural heritage data resource is a valid choice with long-term potential. The evolution of the marketplace and the technology will show which other kind of resources will also appear that have the same potential.

\section{CONCLUSIONS}

Images of 3D models are used frequently in all domains, including cultural heritage. Providing 3D models to a general public however is new and cannot be done without clear goals and methodology.

We have opted here for encapsulating 3D models in a PDF file, as this format is known by nearly every Internet user, a large majority of the Internet users has a PDF reader already installed and it is available and stable on all major platforms. PDF is a standardised and open format, with several software companies providing authoring systems and readers. 3D models from a large variety of formats can be translated easily into PDF, with all required information and files (shape information, textures, materials, ...) encapsulated inside the PDF file, which provides a robust and easy way of delivering $3 \mathrm{D}$ data.

PDF also provides a large set of possibilities to create VR (3D plus behaviour) to improve the exploration and learning capabilities of the 3D data. It provides also tools for collaboration and knowledge exchange. This makes PDF a suitable platform for education and tourism, but allows also developing professional applications in 3D for research, training, restoration, site management in cultural heritage.

3D in Europeana, as provided by CARARE, distinguishes itself from other 3D repositories by the quality of the 3D data and its metadata, providing a reliable source of information and allowing a much better resource exploration. 3D PDF files can incorporate additional information to the 3D (such as text, photographs, iconography, URLs, ...) to improve the exploration of the 3D resource (especially when it depicts explorable spaces) but also to produce unique resources for education, tourism and research. The link between text and 3D (as demonstrated by the examples above in this text) provides a VR behaviour that is easy to create but also yields interactive $3 \mathrm{D}$ with a near to zero learning curve that will appeal to all users of Europeana. We hope that in this way, the uptake of 3D cultural resources through Europeana will be easy and smooth for all users, from children to experts.

\section{ACKNOWLEDGEMENTS}

I would like to thank Sven Havemann (University of Graz) who brought 3D PDF and its use in cultural heritage to my attention. I also want to thank my CARARE colleagues Kate Fernie, Emilia Masci, Sorin Hermon, David Haskiya, Julia Brungs and Ana Martinez for their valuable input and ideas on 3D and Europeana.

CARARE is funded by the European Commission's ICT Policy Support Programme. 


\section{REFERENCES}

AppStorm, 2010. 10 HTML5 Games Paving The Way, http://web.appstorm.net/roundups/browsers/10-html5-gamespaving-the-way/ (accessed 5 Feb 2011)

CATA, 2009. CATA digital reference collection for Iberian pottery, http://cata.cica.es/ (accessed 5 Feb 2011)

Easypano, 2010. ModelWeaver 3.00 to create interactive objects, http://www.easypano.com/modelweaver-gallery.html (accessed 5 Feb 2011)

Müller P., 2007. Nymphaeum: Making of, image gallery, http://www.vision.ee.ethz.ch/ pmueller/wiki/Nymphaeum/Pictu resMakingOf/ (accessed 5 Feb 2011)

Nilsson et al, 2007. ARC 3D Webservice, EPOCH Know How book, http://media.digitalheritage.se/2010/07/ARC_3D_TII.pdf (accessed 5 Feb 2011)

PDF3D, 2010. http://www.pdf3d.com/ (accessed 5 Feb 2011)

Pletinckx D., 2007. Interpretation Management, How to make sustainable visualisations of the past, EPOCH Know How book, http://media.digitalheritage.se/2010/07/Interpretation_Managme nt_TII.pdf (accessed 5 Feb 2011)

Pletinckx, 2008. Interactive Landscapes, How to read and understand historical landscapes, EPOCH Know How book, http://media.digitalheritage.se/2010/07/Interactive_Landscapes_ TII.pdf (accessed 5 Feb 2011)

Rome Reborn, 2010. A Digital Model of Ancient Rome (v. 2.1), http://www.romereborn.virginia.edu/ (accessed 5 Feb 2011)

Unity3D, 2011. http://unity3d.com/ (accessed 5 Feb 2011)

VHLab, 2007. Via Flaminia project (National Museum, Rome) http://www.vhlab.itabc.cnr.it/flaminia/ (accessed 5 Feb 2011) 